\title{
Linear Combinations of Frames and Frame Packets
}

\author{
O. Christensen
}

\begin{abstract}
We find coefficients $c_{m n} \quad(m, n \in \mathbb{Z})$ such that for an arbitrary frame $\left\{f_{n}\right\}_{n \in \mathbb{Z}}$ the set of vectors $\left\{\phi_{m}\right\}_{m \in \mathbb{Z}}=\left\{\sum_{n \in \mathbb{Z}} c_{m n} f_{n}\right\}_{m \in \mathbb{Z}}$ will again be a frame. Appropriate coefficients can always be chosen as function values $c_{m n}=g\left(\frac{n}{\beta}-m \alpha\right)$, where $g$ belongs to a broad class of functions generating a Gabor frame $\left\{E_{\beta m} T_{\alpha n} g\right\}_{m, n \in \mathbb{Z}}$ for $L^{2}(\mathbb{R})$. We also prove a version of the splitting trick, which allows to construct a large family of frames based on a single (wavelet or Gabor) frame.
\end{abstract}

Keywords: Frames, frame packets, Gabor frames

AMS subject classification: $42 \mathrm{C} 15$

\section{Introduction}

Let $\mathcal{H}$ be a Hilbert space with inner product $\langle\cdot, \cdot\rangle$ linear in the first entry. An orthonormal basis $\left\{f_{n}\right\}$ (or more generally, a frame) for $\mathcal{H}$ allows every $f \in \mathcal{H}$ to be written as $f=\sum c_{n} f_{n}$ for certain coefficients $c_{n}$. Here we study the following question: given a frame $\left\{f_{n}\right\}$, define a set of functions $\left\{\phi_{m}\right\}$ by taking appropriate linear combinations of the elements $f_{n}$. Under which conditions (on the coefficients in the linear combinations) will $\left\{\phi_{m}\right\}$ also be a frame? We find a sufficient condition for $\left\{\phi_{m}\right\}$ to be a frame, which is independent of the given frame $\left\{f_{n}\right\}$. Suitable coefficients can be constructed as function values of the "Gabor atom" associated to a broad class of Gabor frames. We also prove a version of the splitting trick, which allows us to construct a large family of frames based on a single (wavelet or) Gabor frame. The fact that our coefficients are independent of $\left\{f_{n}\right\}$ makes succesive applications of the splitting trick very easy, because the same coefficients can be applied repeatedly.

In the rest of this introduction we collect some definitions and basic results that will be used throughout the paper.

\section{Definition 1.1.}

(a) A countable family $\left\{f_{n}\right\}_{n \in I} \subseteq \mathcal{H}$ is a frame for $\mathcal{H}$ if there exist constants $A, B>0$ such that

$$
A\|f\|^{2} \leq \sum_{n \in I}\left|\left\langle f, f_{n}\right\rangle\right|^{2} \leq B\|f\|^{2}
$$

for all $f \in \mathcal{H}$. Numbers $A$ and $B$ that can be used in (1) are called frame bounds.

O. Christensen: Tech. Univ. of Denmark, Dept. Math., Building 303, 2800 Lyngby, Denmark Ole.Christensen@mat.dtu.dk 
(b) $\left\{f_{n}\right\}_{n \in I} \subseteq \mathcal{H}$ is a Riesz basis for $\mathcal{H}$ if $\overline{\operatorname{span}}\left\{f_{n}\right\}_{n \in I}=\mathcal{H}$ and there exists constants $A, B>0$ such that

$$
A \sum\left|c_{n}\right|^{2} \leq\left\|\sum c_{n} f_{n}\right\|^{2} \leq B \sum\left|c_{n}\right|^{2}
$$

for all finite scalar sequences $\left\{c_{n}\right\}$.

If $\left\{f_{n}\right\}_{n \in I}$ is a frame (resp. Riesz basis) for $\overline{\operatorname{span}}\left\{f_{n}\right\}_{n \in I}$, we call $\left\{f_{n}\right\}_{n \in I}$ a frame sequence (resp. Riesz sequence). A Riesz sequence is a frame sequence, and the numbers $A$ and $B$ in (2) are frame bounds.

If $\left\{f_{n}\right\}_{n \in I}$ is a frame for $\mathcal{H}$, we can define a bounded operator

$$
T: \ell^{2}(I) \rightarrow \mathcal{H}, \quad T\left\{c_{n}\right\}_{n \in I}=\sum_{n \in I} c_{n} f_{n}
$$

By composing $T$ with its adjoint, we obtain an invertible operator $T T^{*}$ on $\mathcal{H}$, a fact that leads to a representation of each $f \in \mathcal{H}$ as a (infinite) linear combination of the frame elements (cf. [8]):

$$
f=\sum_{n \in I}\left\langle f,\left(T T^{*}\right)^{-1} f_{n}\right\rangle f_{n}
$$

This formula makes it natural to think about frames as some kind of "generalized basis". Most frames of practical use are coherent, i.e. they appear by letting an appropriate family of operators act on a fixed function. We will consider the operators defined on functions $f \in L^{2}(\mathbb{R})$ by

translation by $\alpha \in \mathbb{R}: \quad\left(T_{\alpha} f\right)(x)=f(x-\alpha)$

modulation by $\beta \in \mathbb{R}: \quad\left(E_{\beta} f\right)(x)=e^{2 \pi i \beta x} f(x)$.

We shall consider frames of the type

- $\left\{T_{\alpha n} f\right\}_{n \in \mathbb{Z}}$ for subspaces of $L^{2}(\mathbb{R})$

- $\left\{E_{\beta m} T_{\alpha n} f\right\}_{m, n \in \mathbb{Z}}$ for $L^{2}(\mathbb{R})$ (Gabor frames).

The Fourier transform is defined by

$$
\hat{f}(\gamma)=\int_{\mathbb{R}} f(x) e^{-2 \pi i \gamma x} d x
$$

With this definition of the Fourier transform, we obtain

$$
\left(T_{\alpha} f\right)^{\wedge}(\gamma)=e^{-2 \pi i \gamma \alpha} \hat{f}(\gamma)
$$

Given $f \in L^{2}(\mathbb{R})$ and a parameter $\alpha>0$, define

$$
F_{\alpha}(\gamma)=\sum_{n \in \mathbb{Z}}\left|\hat{f}\left(\frac{\gamma+n}{\alpha}\right)\right|^{2} .
$$

We refer to $[2,4]$ for a proof of the statement below:

Lemma 1.2. Let $f \in L^{2}(\mathbb{R})$ and let $\alpha$ be a positive real number. Then:

(i) $\left\{T_{\alpha n} f\right\}_{n \in \mathbb{Z}}$ is a Riesz sequence with bounds $A$ and $B$ if and only if $\alpha A \leq$ $F_{\alpha}(\gamma) \leq \alpha B$ for a.e. $\gamma \in[0,1]$.

(ii) $\left\{T_{\alpha n} f\right\}_{n \in \mathbb{Z}}$ is a frame sequence with bounds $A$ and $B$ if and only if $\alpha A \leq$ $F_{\alpha}(\gamma) \leq \alpha B$ for a.e. $\gamma \in[0,1] \backslash\left\{\gamma: F_{\alpha}(\gamma)=0\right\}$. 


\section{Frames formed by linear combination}

Let $\left\{f_{n}\right\}_{n \in I}$ be a frame for $\mathcal{H}$. Suppose that for each $m$ in a countable index set $J$, a set of coefficients $\left\{c_{m n}\right\}_{n \in I} \subseteq \ell^{2}(I)$ is given, and set

$$
\phi_{m}=\sum_{n \in I} c_{m n} f_{n} .
$$

Assume at the moment that $I=J=\mathbb{Z}$. Aldroubi [1] proved that if the matrix $\left\{c_{m n}\right\}_{m, n \in \mathbb{Z}}$ defines a bounded operator on $\ell^{2}(\mathbb{Z})$, then $\left\{\phi_{m}\right\}_{m \in \mathbb{Z}}$ is a frame for $\mathcal{H}$ if and only if there exists a constant $\gamma>0$ such that

$$
\sum_{m \in \mathbb{Z}}\left|\sum_{n \in \mathbb{Z}}\left\langle f, f_{n}\right\rangle c_{m n}\right|^{2} \geq \gamma^{2} \sum_{n \in \mathbb{Z}}\left|\left\langle f, f_{n}\right\rangle\right|^{2}
$$

for all $f \in \mathcal{H}$. In practice, it is difficult to check condition (6). Below we present a sufficient condition for $\left\{\phi_{m}\right\}$ to be a frame which is easier to work with. It can be applied for frames with an arbitrary countable index set, so for later reference we state it in full generality.

Theorem 2.1. Suppose that $\left\{f_{n}\right\}_{n \in I}$ is a Bessel sequence. If

$$
\begin{aligned}
c & :=\inf _{n \in I}\left[\sum_{m \in J}\left|c_{m n}\right|^{2}-\sum_{k \neq n}\left|\sum_{m \in J} c_{m n} \overline{c_{m k}}\right|\right]>0 \\
d & :=\sup _{n \in I} \sum_{k \in I}\left|\sum_{m \in J} c_{m n} \overline{c_{m k}}\right|<\infty,
\end{aligned}
$$

then $\left\{\phi_{m}\right\}_{m \in J}$ defined by (5) satisfies

$$
c \sum_{n \in I}\left|\left\langle f, f_{n}\right\rangle\right|^{2} \leq \sum_{m \in J}\left|\left\langle f, \phi_{m}\right\rangle\right|^{2} \leq d \sum_{n \in I}\left|\left\langle f, f_{n}\right\rangle\right|^{2}
$$

for all $f \in \mathcal{H}$. In particular, if $\left\{f_{n}\right\}_{n \in I}$ is a frame for $\mathcal{H}$ with bounds $C$ and $D$, then $\left\{\phi_{m}\right\}_{m \in J}$ is a frame for $\mathcal{H}$ with bounds $c C$ and $d D$.

Proof. Let $f \in \mathcal{H}$. Then

$$
\begin{aligned}
\sum_{m}\left|\left\langle\phi_{m}, f\right\rangle\right|^{2} & =\sum_{m}\left|\left\langle\sum_{n} c_{m n} f_{n}, f\right\rangle\right|^{2} \\
& =\sum_{m}\left|\sum_{n} c_{m n}\left\langle f_{n}, f\right\rangle\right|^{2} \\
& =\sum_{m} \sum_{n}\left|c_{m n}\right|^{2} \cdot\left|\left\langle f_{n}, f\right\rangle\right|^{2}+\sum_{m} \sum_{n} \sum_{k \neq n} c_{m n} \overline{c_{m k}}\left\langle f_{n}, f\right\rangle\left\langle f, f_{k}\right\rangle \\
& =:(*)+(* *) .
\end{aligned}
$$


By Cauchy-Schwarz' inequality we get

$$
\begin{aligned}
|(* *)| & \leq \sum_{n} \sum_{k \neq n}\left|\left\langle f_{n}, f\right\rangle\left\langle f, f_{k}\right\rangle\right| \cdot\left|\sum_{m} c_{m n} \overline{c_{m k}}\right| \\
& \leq\left(\sum_{n} \sum_{k \neq n}\left|\left\langle f_{n}, f\right\rangle\right|^{2} \cdot\left|\sum_{m} c_{m n} \overline{c_{m k}}\right|\right)^{\frac{1}{2}}\left(\sum_{n} \sum_{k \neq n}\left|\left\langle f, f_{k}\right\rangle\right|^{2} \cdot\left|\sum_{m} c_{m n} \overline{c_{m k}}\right|\right)^{\frac{1}{2}} .
\end{aligned}
$$

The two terms in the last product are actually identical, since

$$
\begin{aligned}
\sum_{n} \sum_{k \neq n}\left|\left\langle f, f_{k}\right\rangle\right|^{2} \cdot\left|\sum_{m} c_{m n} \overline{c_{m k}}\right| & =\sum_{k} \sum_{n \neq k}\left|\left\langle f, f_{k}\right\rangle\right|^{2} \cdot\left|\sum_{m} c_{m n} \overline{c_{m k}}\right| \\
& =\sum_{n} \sum_{k \neq n}\left|\left\langle f_{n}, f\right\rangle\right|^{2} \cdot\left|\sum_{m} c_{m n} \overline{c_{m k}}\right| .
\end{aligned}
$$

Thus

$$
(* *) \leq \sum_{n} \sum_{k \neq n}\left|\left\langle f_{n}, f\right\rangle\right|^{2} \cdot\left|\sum_{m} c_{m n} \overline{c_{m k}}\right|
$$

and, by the calculation at the beginning of the proof,

$$
\begin{aligned}
\sum_{m}\left|\left\langle\phi_{m}, f\right\rangle\right|^{2} & \geq \sum_{m} \sum_{n}\left|c_{m n}\right|^{2} \cdot\left|\left\langle f_{n}, f\right\rangle\right|^{2}-\sum_{n} \sum_{k \neq n}\left|\left\langle f_{n}, f\right\rangle\right|^{2} \cdot\left|\sum_{m} c_{m n} \overline{c_{m k}}\right| \\
& =\sum_{n}\left|\left\langle f_{n}, f\right\rangle\right|^{2} \cdot\left[\sum_{m}\left|c_{m n}\right|^{2}-\sum_{k \neq n}\left|\sum_{m} c_{m n} \overline{c_{m k}}\right|\right] \\
& \geq c \sum_{n}\left|\left\langle f_{n}, f\right\rangle\right|^{2} .
\end{aligned}
$$

The upper frame condition is proved similarly

Note that the condition in Theorem 2.1 is independent of $\left\{f_{n}\right\}$. In order to give a geometric interpretation of the condition, suppose that $\left\{f_{n}\right\}$ and $\left\{\phi_{m}\right\}$ are indexed by $\mathbb{Z}$. Think about the sequence $\left\{c_{m n}\right\}_{m, n \in \mathbb{Z}}$ as a matrix, where $m$ is the row index and $n$ the column index:

$$
\left\{c_{m n}\right\}_{m, n \in \mathbb{Z}}=\left(\begin{array}{ccccc}
\cdot & \cdot & \cdot & \cdot & \cdot \\
\cdot & c_{-1-1} & c_{-10} & c_{-11} & \cdot \\
\cdot & c_{0-1} & c_{00} & c_{01} & \cdot \\
\cdot & c_{1-1} & c_{10} & c_{11} & \cdot \\
\cdot & \cdot & \cdot & \cdot & \cdot
\end{array}\right)
$$

For $n$ fixed, the sum $\sum_{m \in \mathbb{Z}}\left|c_{m n}\right|^{2}$ is equal to the scalar product in $\ell^{2}(\mathbb{Z})$ of the $n$ 'th column with itself, while $\sum_{k \neq n}\left|\sum_{m \in \mathbb{Z}} c_{m n} \overline{c_{m k}}\right|$ is the sum of the absolute values of the scalar products between the $n$ 'th column and all other columns $k$. In particular, in order for Theorem 2.1 to apply no column can consist solely of zeros (zero-rows might exist, but they are not interesting, since they correspond to a $\phi_{m}$ defined to be zero). The remark clearly holds for arbitrarily indexed sets. 
Example 2.2. Let $\left\{e_{n}\right\}_{n=1}^{\infty}$ be an orthonormal basis. For a given number $\alpha \geq 0$, define

$$
\phi_{m}=e_{m}+\alpha e_{m+1} \quad(m \in \mathbb{N}) .
$$

In the notation above (with $\mathbb{Z}$ replaced by $\mathbb{N}$ ) we have

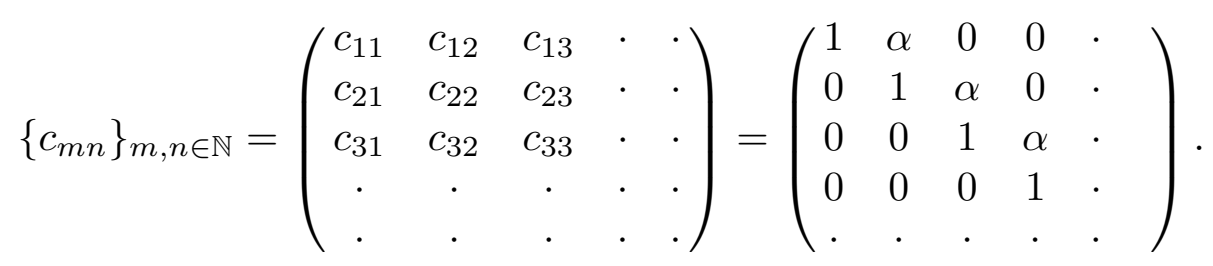

It is an easy consequence of Theorem 2.1 that $\left\{\phi_{m}\right\}_{m=1}^{\infty}$ is a frame if $\alpha<1$. This is actually the optimal conclusion: $\left\{\phi_{m}\right\}_{m=1}^{\infty}$ is not a frame if $\alpha \geq 1$.

It is easy to describe a general class of coefficients that can be used in Theorem 2.1. We use a general sufficient condition for $\left\{E_{\beta m} T_{\alpha n} g\right\}_{m, n \in \mathbb{Z}}$ to be a frame for $L^{2}(\mathbb{R})$ (cf. [3] or [9]):

Lemma 2.3. Let $g \in L^{2}(\mathbb{R}), \quad \alpha, \beta>0$ and suppose that there exist numbers $A, B>0$ such that for all $x \in \mathbb{R}$

$$
\begin{array}{r}
\sum_{m \in \mathbb{Z}}|g(x-m \alpha)|^{2}-\sum_{k \neq 0}\left|\sum_{m \in \mathbb{Z}} g(x-m \alpha) \overline{g\left(x-m \alpha-\frac{k}{\beta}\right)}\right| \geq A \\
\sum_{k \in \mathbb{Z}}\left|\sum_{m \in \mathbb{Z}} g(x-m \alpha) \overline{g\left(x-m \alpha-\frac{k}{\beta}\right)}\right| \leq B .
\end{array}
$$

Then $\left\{E_{\beta m} T_{\alpha n} g\right\}_{m, n \in \mathbb{Z}}$ is a frame for $L^{2}(\mathbb{R})$ with bounds $\frac{A}{\beta}$ and $\frac{B}{\beta}$.

Lemma 2.3 actually holds if (8) - (9) are satisfied for almost all $x \in \mathbb{R}$, but for our application below it is essential to assume the conditions satisfied for all $x$.

Theorem 2.4. Let $\left\{f_{n}\right\}_{n \in \mathbb{Z}}$ be a frame with bounds $C$ and D. Let $\left\{E_{\beta m} T_{\alpha n} g\right\}_{m, n \in \mathbb{Z}}$ be a frame satisfying (8) - (9), and define

$$
\phi_{m}=\sum_{n \in \mathbb{Z}} c_{m n} f_{n}, \quad \text { where } c_{m n}=g\left(\frac{n}{\beta}-m \alpha\right) .
$$

Then $\left\{\phi_{m}\right\}_{m \in \mathbb{Z}}$ is a frame with bounds $A C$ and $B D$.

Proof. Let $n \in \mathbb{Z}$. Using condition (8) on $x=\frac{n}{\beta}$ gives

$$
\sum_{m \in \mathbb{Z}}\left|g\left(\frac{n}{\beta}-m \alpha\right)\right|^{2}-\sum_{k \neq 0}\left|\sum_{m \in \mathbb{Z}} g\left(\frac{n}{\beta}-m \alpha\right) \overline{g\left(\frac{n}{\beta}-m \alpha-\frac{k}{\beta}\right)}\right| \geq A .
$$

By the change of variable $k \rightarrow n-k$,

$$
\sum_{m \in \mathbb{Z}}\left|g\left(\frac{n}{\beta}-m \alpha\right)\right|^{2}-\sum_{k \neq n}\left|\sum_{m \in \mathbb{Z}} g\left(\frac{n}{\beta}-m \alpha\right) \overline{g\left(\frac{k}{\beta}-m \alpha\right)}\right| \geq A .
$$


Similarly,

$$
\sum_{k \in \mathbb{Z}}\left|\sum_{m \in \mathbb{Z}} g\left(\frac{n}{\beta}-m \alpha\right) \overline{g\left(\frac{k}{\beta}-m \alpha\right)}\right| \leq B
$$

for all $n \in \mathbb{Z}$. It follows that the coefficients $c_{m n}=g\left(\frac{n}{\beta}-m \alpha\right)$ satisfy the conditions in Theorem 2.1 with $c=A$ and $d=B$. Thus $\left\{\phi_{m}\right\}_{m \in \mathbb{Z}}$ is a frame with bounds $A C$ and $B D$

For frames of translates the general condition in Theorem 2.1 simplifies:

Corollary 2.5. Suppose that $\left\{T_{\alpha n} f\right\}_{n \in \mathbb{Z}}$ is a frame for $V=\overline{\operatorname{span}}\left\{T_{\alpha n} f\right\}_{n \in \mathbb{Z}}$ with bounds $A$ and $B$. Given a set of coefficients $\left\{c_{n}\right\} \in \ell^{2}(\mathbb{Z})$, set $\phi=\sum_{n \in \mathbb{Z}} c_{n} T_{\alpha n} f$. If

$$
\begin{aligned}
c & :=\sum_{m \in \mathbb{Z}}\left|c_{m}\right|^{2}-\sum_{k \neq 0}\left|\sum_{m \in \mathbb{Z}} c_{m} \overline{c_{m+k}}\right|>0 \\
d & :=\sum_{k \in \mathbb{Z}}\left|\sum_{m \in \mathbb{Z}} c_{m} \overline{c_{m+k}}\right|<\infty,
\end{aligned}
$$

then $\left\{T_{\alpha m} \phi\right\}_{m \in \mathbb{Z}}$ is a frame for $V$ with bounds $c A$ and $d B$.

Proof. We have

$$
T_{\alpha m} \phi=\sum_{n \in \mathbb{Z}} c_{n} T_{\alpha(n+m)} f=\sum_{n \in \mathbb{Z}} c_{n-m} T_{\alpha n} f .
$$

We now use Theorem 2.1 with $c_{m n}=c_{n-m}$. Given $n \in \mathbb{Z}$,

$$
\begin{aligned}
\sum_{m \in \mathbb{Z}}\left|c_{m n}\right|^{2}-\sum_{k \neq n}\left|\sum_{m \in \mathbb{Z}} c_{m n} \overline{c_{m k}}\right| & =\sum_{m \in \mathbb{Z}}\left|c_{n-m}\right|^{2}-\sum_{k \neq n}\left|\sum_{m \in \mathbb{Z}} c_{n-m} \overline{c_{k-m}}\right| \\
& =\sum_{m \in \mathbb{Z}}\left|c_{m}\right|^{2}-\sum_{k \neq 0}\left|\sum_{m \in \mathbb{Z}} c_{m} \overline{c_{k+m}}\right|
\end{aligned}
$$

which is independent of $n$. The upper condition follows similarly

\section{The splitting trick}

The splitting trick (introduced by Daubechies [7]) was originally used to construct a large family of wavelet bases (called a wavelet packet) starting with a single basis. Later similar techniques have been applied to wavelet frames [5]. Here we show that the results in the previous sections lead to versions of the splitting trick that are very easy to apply.

Given a sequence $\left\{c_{n}\right\}_{n \in \mathbb{Z}} \subseteq \ell^{2}(\mathbb{Z})$, we define the associated two-scale symbol as the function

$$
m(\gamma)=\frac{1}{\sqrt{2}} \sum_{n \in \mathbb{Z}} c_{n} e^{-2 \pi i \gamma n} \quad(\gamma \in \mathbb{R}) .
$$

Note that $m \in L^{2}(0,1)$ and that $m$ has period 1 .

The basic idea in the splitting trick is to split a frame $\left\{T_{\alpha n} f\right\}_{n \in \mathbb{Z}}$ with translation parameter $\alpha$ into the union of two families with translation parameter $2 \alpha$. We start with some lemmas. 
Lemma 3.1. Suppose that $\left\{T_{\alpha n} f\right\}_{n \in \mathbb{Z}}$ is a Riesz sequence with bounds $A$ and $B$. Given a sequence $\left\{c_{n}\right\}_{n \in \mathbb{Z}} \in \ell^{2}(\mathbb{Z})$, let $m$ denote the associated two-scale symbol and suppose that there exist constants $a, b>0$ such that

$$
a \leq|m(\gamma)|^{2}+\left|m\left(\gamma+\frac{1}{2}\right)\right|^{2} \leq b
$$

for a.e. $\gamma \in[0,1]$. Set $\phi=\sum_{n \in \mathbb{Z}} c_{n} T_{\alpha n} f$. Then $\left\{T_{2 \alpha m} \phi\right\}_{m \in \mathbb{Z}}$ is a Riesz sequence with bounds $a A$ and $b B$.

Proof. In order to apply Lemma 1.2, let $\Phi_{2 \alpha}(\gamma)=\sum_{n \in \mathbb{Z}}\left|\hat{\phi}\left(\frac{\gamma+n}{2 \alpha}\right)\right|^{2}$. Splitting the sum into two and applying (3), we get

$$
\begin{aligned}
\Phi_{2 \alpha}(\gamma)= & \sum_{n \in \mathbb{Z}}\left|\hat{\phi}\left(\frac{\gamma}{2 \alpha}+\frac{n}{\alpha}\right)\right|^{2}+\sum_{n \in \mathbb{Z}}\left|\hat{\phi}\left(\frac{\gamma}{2 \alpha}+\frac{1}{2 \alpha}+\frac{n}{\alpha}\right)\right|^{2} \\
= & 2 \sum_{n \in \mathbb{Z}}\left|m\left(\frac{\gamma}{2}+n\right)\right|^{2}\left|\hat{f}\left(\frac{\gamma}{2 \alpha}+\frac{n}{\alpha}\right)\right|^{2} \\
& +2 \sum_{n \in \mathbb{Z}}\left|m\left(\frac{\gamma}{2}+\frac{1}{2}+n\right)\right|^{2}\left|\hat{f}\left(\frac{\gamma}{2 \alpha}+\frac{1}{2 \alpha}+\frac{n}{\alpha}\right)\right|^{2} \\
= & 2\left|m\left(\frac{\gamma}{2}\right)\right|^{2} \sum_{n \in \mathbb{Z}}\left|\hat{f}\left(\frac{\gamma}{2 \alpha}+\frac{n}{\alpha}\right)\right|^{2}+2\left|m\left(\frac{\gamma}{2}+\frac{1}{2}\right)\right|^{2} \sum_{n \in \mathbb{Z}}\left|\hat{f}\left(\frac{\gamma}{2 \alpha}+\frac{1}{2 \alpha}+\frac{n}{\alpha}\right)\right|^{2} .
\end{aligned}
$$

By Lemma 1.2(i) we have $\alpha A \leq \sum_{n \in \mathbb{Z}}\left|\hat{f}\left(\frac{\gamma+n}{\alpha}\right)\right|^{2} \leq \alpha B$ for a.e. $\gamma \in[0,1]$. Thus, for a.e. $\gamma$ the calculation above gives $2 a \alpha A \leq \Phi_{2 \alpha}(\gamma) \leq 2 b \alpha B$. Now the conclusion follows from Lemma 1.2(i)

Lemma 3.1 does not extend to frame sequences, i.e. if $\left\{T_{\alpha n} f\right\}_{n \in \mathbb{Z}}$ is only assumed to be a frame sequence, condition (10) does not imply that $\left\{T_{2 \alpha m} \phi\right\}_{m \in \mathbb{Z}}$ is a frame sequence:

Example 3.2. Define $f \in L^{2}(\mathbb{R})$ by $\hat{f}=\chi_{\left[0, \frac{1}{4}\right]}$. Then for $\gamma \in[0,1]$

$$
\sum_{n \in \mathbb{Z}}|\hat{f}(\gamma+n)|^{2}=\chi_{\left[0, \frac{1}{4}\right]}(\gamma)
$$

implying by Lemma 1.2 that $\left\{T_{n} f\right\}_{n \in \mathbb{Z}}$ is a frame sequence with bounds $A=B=1$. Also, for $\gamma \in[0,1]$,

$$
\sum_{n \in \mathbb{Z}}\left|\hat{f}\left(\frac{\gamma}{2}+n\right)\right|^{2}=\left|\hat{f}\left(\frac{\gamma}{2}\right)\right|^{2}=\chi_{\left[0, \frac{1}{2}\right]}(\gamma)
$$

while $\sum_{n \in \mathbb{Z}}\left|\hat{f}\left(\frac{\gamma}{2}+\frac{1}{2}+n\right)\right|^{2}=0$. Now choose $\left\{c_{n}\right\}_{n \in \mathbb{Z}} \in \ell^{2}(\mathbb{Z})$ such that the associated two-scale symbol is given by

$$
m(\gamma)= \begin{cases}\gamma & \text { for } \gamma \in\left[0, \frac{1}{2}\right) \\ \sqrt{1-\left(\gamma-\frac{1}{2}\right)^{2}} & \text { for } \gamma \in\left[\frac{1}{2}, 1\right] .\end{cases}
$$


It is easy to check that for $\gamma \in[0,1)$ we have $|m(\gamma)|^{2}+\left|m\left(\gamma+\frac{1}{2}\right)\right|^{2}=1$. As in Lemma 3.1 , let $\phi=\sum_{n \in \mathbb{Z}} c_{n} T_{n} f$. By the calculation in the proof of Lemma 3.1, for $\gamma \in\left[0, \frac{1}{2}\right)$ we have

$$
\begin{aligned}
\Phi_{2}(\gamma) & =\sum_{n \in \mathbb{Z}}\left|\hat{\phi}\left(\frac{\gamma+n}{2}\right)\right|^{2} \\
& =2\left|m\left(\frac{\gamma}{2}\right)\right|^{2} \sum_{n \in \mathbb{Z}}\left|\hat{f}\left(\frac{\gamma}{2}+n\right)\right|^{2}+2\left|m\left(\frac{\gamma}{2}+\frac{1}{2}\right)\right|^{2} \sum_{n \in \mathbb{Z}}\left|\hat{f}\left(\frac{\gamma}{2}+\frac{1}{2}+n\right)\right|^{2} \\
& =2\left|m\left(\frac{\gamma}{2}\right)\right|^{2} \\
& =\frac{\gamma^{2}}{2} .
\end{aligned}
$$

Since $\Phi_{2}$ is not bounded away from zero, we conclude by Lemma 1.2(ii) that $\left\{T_{2 m} \phi\right\}_{m \in \mathbb{Z}}$ is not a frame sequence

However, a different condition on $m(\gamma)$ leads to a version of Lemma 3.1 which can be used for frame sequences:

Lemma 3.3. Suppose that $\left\{T_{\alpha n} f\right\}_{n \in \mathbb{Z}}$ is a frame sequence with bounds $A$ and $B$. Given a sequence $\left\{c_{n}\right\}_{n \in \mathbb{Z}} \in \ell^{2}(\mathbb{Z})$, suppose that the associated two-scale symbol $m$ satisfies $0<a \leq|m(\gamma)|^{2} \leq b$ for a.e. $\gamma \in[0,1]$ and set $\phi=\sum_{n \in \mathbb{Z}} c_{n} T_{\alpha n} f$. Then $\left\{T_{2 \alpha m} \phi\right\}_{m \in \mathbb{Z}}$ is a frame sequence with bounds $a A$ and $2 b B$.

Proof. The definition of $\phi$ implies $\hat{\phi}(\gamma)=\sqrt{2} m(\gamma \alpha) \hat{f}(\gamma)$. Therefore, with notation (4),

$$
\begin{aligned}
\Phi_{2 \alpha}(\gamma)= & \sum_{n \in \mathbb{Z}}\left|\hat{\phi}\left(\frac{\gamma+n}{2 \alpha}\right)\right|^{2} \\
= & \sum_{n \in \mathbb{Z}}\left|\hat{\phi}\left(\frac{\gamma+2 n}{2 \alpha}\right)\right|^{2}+\sum_{n \in \mathbb{Z}}\left|\hat{\phi}\left(\frac{\gamma+2 n+1}{2 \alpha}\right)\right|^{2} \\
= & \sum_{n \in \mathbb{Z}}\left|\hat{\phi}\left(\frac{\gamma}{2 \alpha}+\frac{n}{\alpha}\right)\right|^{2}+\sum_{n \in \mathbb{Z}}\left|\hat{\phi}\left(\frac{\gamma}{2 \alpha}+\frac{1}{2 \alpha}+\frac{n}{\alpha}\right)\right|^{2} \\
= & 2 \cdot \sum_{n \in \mathbb{Z}}\left|m\left(\frac{\gamma}{2}+n\right)\right|^{2} \cdot\left|\hat{f}\left(\frac{\gamma}{2 \alpha}+\frac{n}{\alpha}\right)\right|^{2} \\
& +2 \cdot \sum_{n \in \mathbb{Z}}\left|m\left(\frac{\gamma}{2}+\frac{1}{2}+n\right)\right|^{2} \cdot\left|\hat{f}\left(\frac{\gamma}{2 \alpha}+\frac{1}{2 \alpha}+\frac{n}{\alpha}\right)\right|^{2} \\
= & 2 \cdot\left|m\left(\frac{\gamma}{2}\right)\right|^{2} \sum_{n \in \mathbb{Z}}\left|\hat{f}\left(\frac{\gamma}{2 \alpha}+\frac{n}{\alpha}\right)\right|^{2} \\
& +2 \cdot\left|m\left(\frac{\gamma}{2}+\frac{1}{2}\right)\right|^{2} \sum_{n \in \mathbb{Z}}\left|\hat{f}\left(\frac{\gamma}{2 \alpha}+\frac{1}{2 \alpha}+\frac{n}{\alpha}\right)\right|^{2} \cdot
\end{aligned}
$$

With $F_{\alpha}(\gamma)=\sum_{n \in \mathbb{Z}}\left|\hat{f}\left(\frac{\gamma+n}{\alpha}\right)\right|^{2}$ we have $\alpha A \leq F_{\alpha}(\gamma) \leq B \alpha$ except on the zero set for 
$F_{\alpha}$. Thus $\Phi_{2 \alpha}(\gamma) \leq 4 b B \alpha$ a.e. Set now

$$
\begin{aligned}
N & =\left\{\gamma: \Phi_{2 \alpha}(\gamma)=0\right\} \\
& =\left\{\gamma: \sum_{n \in \mathbb{Z}}\left|\hat{f}\left(\frac{\frac{\gamma+1}{2}+n}{\alpha}\right)\right|^{2}=0 \text { and } \sum_{n \in \mathbb{Z}}\left|\hat{f}\left(\frac{\frac{\gamma}{2}+n}{\alpha}\right)\right|^{2}=0\right\} .
\end{aligned}
$$

For a.e. $\gamma \notin N$ we have $\Phi_{2 \alpha}(\gamma) \geq 2 a A \alpha=2 \alpha a A$. Thus $\left\{T_{2 \alpha m} \phi\right\}_{m \in \mathbb{Z}}$ is a frame sequence with bounds $a A$ and $2 b B$

We now present a version of the "splitting trick".

Theorem 3.4. Suppose $\left\{T_{\alpha n} f\right\}_{n \in \mathbb{Z}}$ is a frame for $V=\overline{\operatorname{span}}\left\{T_{\alpha n} f\right\}_{n \in \mathbb{Z}}$ with bounds $A$ and $B$. Let $\left\{c_{n}\right\},\left\{d_{n}\right\} \in \ell^{2}(\mathbb{Z})$ and assume that

$$
\begin{aligned}
a:=\min & \left(\sum_{m \in \mathbb{Z}}\left|c_{2 m}\right|^{2}-\sum_{k \neq 0}\left|\sum_{m \in \mathbb{Z}} c_{2 m} \overline{c_{2 m+k}}\right|\right. \\
& +\sum_{m \in \mathbb{Z}}\left|d_{2 m}\right|^{2}-\sum_{k \neq 0}\left|\sum_{m \in \mathbb{Z}} d_{2 m} \overline{d_{2 m+k}}\right|, \\
& \sum_{m \in \mathbb{Z}}\left|c_{2 m+1}\right|^{2}-\sum_{k \neq 0}\left|\sum_{m \in \mathbb{Z}} c_{2 m+1} \overline{c_{2 m+1+k}}\right| \\
& \left.+\sum_{m \in \mathbb{Z}}\left|d_{2 m+1}\right|^{2}-\sum_{k \neq 0}\left|\sum_{m \in \mathbb{Z}} d_{2 m+1} \overline{d_{2 m+1+k}}\right|\right) \\
>0 & \max \\
& \left(\sum_{k \in \mathbb{Z}}\left|\sum_{m \in \mathbb{Z}} c_{2 m} \overline{c_{2 m+k}}\right|+\sum_{k \in \mathbb{Z}}\left|\sum_{m \in \mathbb{Z}} d_{2 m} \overline{d_{2 m+k}}\right|,\right. \\
& \left.\sum_{k \in \mathbb{Z}}\left|\sum_{m \in \mathbb{Z}} c_{2 m+1} \overline{c_{2 m+1+k}}\right|+\sum_{k \in \mathbb{Z}}\left|\sum_{m \in \mathbb{Z}} d_{2 m+1} \overline{d_{2 m+1+k}}\right|\right) \\
<\infty . &
\end{aligned}
$$

Further, set $\phi_{1}=\sum_{n \in \mathbb{Z}} c_{n} T_{\alpha n} f$ and $\phi_{2}=\sum_{n \in \mathbb{Z}} d_{n} T_{\alpha n} f$. Then, for all $g \in V$,

$$
a \sum_{n \in \mathbb{Z}}\left|\left\langle g, T_{\alpha n} f\right\rangle\right|^{2} \leq \sum_{i=1}^{2} \sum_{m \in \mathbb{Z}}\left|\left\langle g, T_{2 \alpha m} \phi_{i}\right\rangle\right|^{2} \leq b \sum_{n \in \mathbb{Z}}\left|\left\langle g, T_{\alpha n} f\right\rangle\right|^{2} .
$$

In particular, if $\left\{T_{\alpha n} f\right\}_{n \in \mathbb{Z}}$ is a frame for $V$ with bounds $A$ and $B$, then $\left\{T_{2 \alpha m} \phi_{1}\right\}_{m \in \mathbb{Z}} \cup$ $\left\{T_{2 \alpha m} \phi_{2}\right\}_{m \in \mathbb{Z}}$ is a frame for $V$ with bounds $a A$ and $b B$.

Proof. Observe that

$$
T_{2 \alpha m} \phi_{1}=\sum_{n \in \mathbb{Z}} c_{n} T_{\alpha(2 m+n)} f=\sum_{n \in \mathbb{Z}} c_{n-2 m} T_{\alpha n} f
$$


Similarly, $T_{2 \alpha m} \phi_{2}=\sum_{n \in \mathbb{Z}} d_{n-2 m} T_{\alpha n} f$. Set $c_{m n}=c_{n-2 m}$ and $d_{m n}=d_{n-2 m}$, and consider the blockmatrix

$$
\begin{aligned}
C & =\left(\begin{array}{l}
\left\{c_{m n}\right\}_{m, n \in \mathbb{Z}} \\
\left\{d_{m n}\right\}_{m, n \in \mathbb{Z}}
\end{array}\right) \\
& =\left(\begin{array}{l}
\left\{c_{n-2 m}\right\}_{m, n \in \mathbb{Z}} \\
\left\{d_{n-2 m}\right\}_{m, n \in \mathbb{Z}}
\end{array}\right) \\
& \left(\begin{array}{cccccccccc}
\cdot & \cdot & \cdot & \cdot & \cdot & \cdot & \cdot & \cdot & \cdot & \cdot \\
\cdot & \cdot & c_{0} & c_{1} & c_{2} & c_{3} & c_{4} & c_{5} & \cdot & \cdot \\
\cdot & \cdot & c_{-2} & c_{-1} & c_{0} & c_{1} & c_{2} & c_{3} & \cdot & \cdot \\
\cdot & \cdot & c_{-4} & c_{-3} & c_{-2} & c_{-1} & c_{0} & c_{1} & \cdot & \cdot \\
\cdot & \cdot & \cdot & \cdot & \cdot & \cdot & \cdot & \cdot & \cdot \\
\cdot & \cdot & \cdot & \cdot & \cdot & \cdot & \cdot & \cdot & \cdot & \cdot \\
\cdot & \cdot & d_{0} & d_{1} & d_{2} & d_{3} & d_{4} & d_{5} & \cdot & \cdot \\
\cdot & \cdot & d_{-2} & d_{-1} & d_{0} & d_{1} & d_{2} & d_{3} & \cdot & \cdot \\
\cdot & \cdot & d_{-4} & d_{-3} & d_{-2} & d_{-1} & d_{0} & d_{1} & \cdot & \cdot \\
\cdot & \cdot & \cdot & \cdot & \cdot & \cdot & \cdot & \cdot & \cdot & \cdot
\end{array}\right) .
\end{aligned}
$$

Due to the special structure of the matrix, the conditions in Theorem 2.1 will be identical for all even values of $n-$ and for all odd values. This gives the condition stated in Theorem 3.4

The advantage of Theorem 3.4 is that the set of coefficients $\left\{c_{n}\right\}$ and $\left\{d_{n}\right\}$ are independent of $\left\{T_{\alpha n} f\right\}_{n \in \mathbb{Z}}$. That is, if we want to apply the splitting trick repeatedly, the same coefficients can be used again.

If the matrix $C$ from the proof of Theorem 3.4 is orthogonal, then $a=b=1$. That is, the splitting preserves the frame bounds. In applications of Theorem 3.4 it is desirable that $\left\{T_{2 \alpha m} \phi_{1}\right\}_{m \in \mathbb{Z}}$ and $\left\{T_{2 \alpha m} \phi_{2}\right\}_{m \in \mathbb{Z}}$ are frame sequences by themselfes. A sufficient condition on the set of coefficients $\left\{c_{n}\right\}$ and $\left\{d_{n}\right\}$ can be found in Lemma 3.3 (or Lemma 3.1 if $\left\{T_{\alpha n} f\right\}_{n \in \mathbb{Z}}$ is a Riesz sequence).

The splitting trick is traditionally used to construct an infinite family of frames based on a single wavelet. We illustrate the principle with Gabor frames.

Example 3.5. Assume that $\left\{E_{\beta m} T_{\alpha n} g\right\}_{m, n \in \mathbb{Z}}$ is a frame for $L^{2}(\mathbb{R})$; this is clearly the case if and only if $\left\{T_{\alpha n} E_{\beta m} g\right\}_{m, n \in \mathbb{Z}}$ is a frame for $L^{2}(\mathbb{R})$. Denote the frame bounds by $A$ and $B$. By defining $g_{m}=E_{\beta m} g$, the later frame can be written $\left\{T_{\alpha n} g_{m}\right\}_{m, n \in \mathbb{Z}}$. For each $m \in \mathbb{Z},\left\{T_{\alpha n} g_{m}\right\}_{n \in \mathbb{Z}}$ is a Bessel sequence. Choose coefficients $\left\{c_{n}\right\}_{n \in \mathbb{Z}}$ and $\left\{d_{n}\right\}_{n \in \mathbb{Z}}$ as in Theorem 3.4 and define for each $m \in \mathbb{Z}$ the functions

$$
\phi_{m}^{1}=\sum_{n \in \mathbb{Z}} c_{n} T_{\alpha n} g_{m} \quad \text { and } \quad \phi_{m}^{2}=\sum_{n \in \mathbb{Z}} d_{n} T_{\alpha n} g_{m}
$$

By (11), $\left\{T_{2 \alpha n} \phi_{m}^{1}\right\}_{m, n \in \mathbb{Z}} \cup\left\{T_{2 \alpha n} \phi_{m}^{2}\right\}_{m, n \in \mathbb{Z}}$ is a frame for $L^{2}(\mathbb{R})$ with bounds $a A$ and $b B$.

Instead of applying the splitting trick for each value of $m$, we can also decide only to apply the splitting trick to some of the functions $g_{m}$, say, for $m$ in an index set $I$, and keep the rest. In this case we obtain a frame of the form

$$
\left\{T_{2 \alpha n} \phi_{m}^{1}\right\}_{n \in \mathbb{Z}, m \in I} \cup\left\{T_{2 \alpha n} \phi_{m}^{2}\right\}_{n \in \mathbb{Z}, m \in I} \cup\left\{E_{\beta m} T_{\alpha n} g\right\}_{n \in \mathbb{Z}, m \in \mathbb{Z} \backslash I} .
$$


All choices together gives an infinite library of frames with frame bounds $\min (1, a) A$ and $\max (1, b) B$.

We can also apply several succesive splittings, i.e. repeat the splitting on $\phi_{m}^{1}, \phi_{m}^{2}$, etc. If we apply at most $L$ succesive splittings, we obtain a frame for $L^{2}(\mathbb{R})$ with bounds $\min (1, a)^{L} A$ and $\max (1, b)^{L} B$.

Thus, a single Gabor frame allows to construct a whole library of Gabor frames. The advantage of Theorem 3.4 is that the same coefficients $\left\{c_{n}\right\}$ and $\left\{d_{n}\right\}$ can be applied to each splitting.

It is interesting to compare Example 3.5 with the known fact that $\left\{E_{\beta m} T_{\alpha n} g\right\}_{m, n \in \mathbb{Z}}$ can only be a frame for $L^{2}(\mathbb{R})$ if $\alpha \beta \leq 1$. Let $\left\{E_{\beta m} T_{\alpha n} g\right\}_{m, n \in \mathbb{Z}}$ be a Gabor frame with $\alpha \beta=1$. By defining $g^{1}=\sum_{n \in \mathbb{Z}} c_{n} T_{\alpha n} g$ and $g^{2}=\sum_{n \in \mathbb{Z}} d_{n} T_{\alpha n} g$, the functions $\phi_{m}^{1}$ and $\phi_{m}^{2}$ in (12) can be written $\phi_{m}^{i}=E_{\beta m} g^{i} \quad(i=1,2)$. That is, the family $\left\{T_{2 \alpha n} E_{\beta m} g^{i}\right\}_{m, n \in \mathbb{Z}, i=1,2}$ is a frame for $L^{2}(\mathbb{R})$, for which the product of the translation and the modulation parameters is two. The price for this to be possible is that we need two generators $g_{1}$ and $g_{2}$ instead of one.

\section{References}

[1] Aldroubi, A.: Portraits of frames. Proc. Amer. Math. Soc. 123 (1995), $1661-1668$.

[2] Benedetto, J. and S. Li: The theory of multiresolution analysis frames and applications to filter banks. Appl. Comp. Harm. Anal. 5 (1998), 389 - 427.

[3] Casazza, P. G. and O. Christensen: Weyl-Heisenberg frames for subspaces of $L^{2}(\mathbb{R})$. Proc. Amer. Math. Soc. 129 (2001), 145 - 154.

[4] Casazza, C., Christensen, O. and N. Kalton: Frames of translates. Coll. Math. 52 (2001), $35-54$.

[5] Chen, D.: On the splitting trick and wavelet frame packets. SIAM J. Math. Anal. 31 (2000), $726-739$.

[6] Christensen, O. and C. Heil: Perturbation of Banach frames and atomic decomposition. Math. Nach. 185 (1997), $33-47$.

[7] Daubechies, I.: Ten lectures on Wavelets (SIAM Conf. Series in Applied Math.). Boston: SIAM 1992.

[8] Heil, C. and D. Walnut: Continuous and discrete wavelet transforms. SIAM Review 31 (1989), $628-666$.

[9] Ron, A. and Z. Shen: Weyl-Heisenberg systems and Riesz bases in $L^{2}\left(\mathbb{R}^{d}\right)$. Duke Math. J. 89 (1997), $237-282$.

Received 01.03.2001 